\section{Quasireform of English Spelling}

Neville Holmes, University of Tasmania

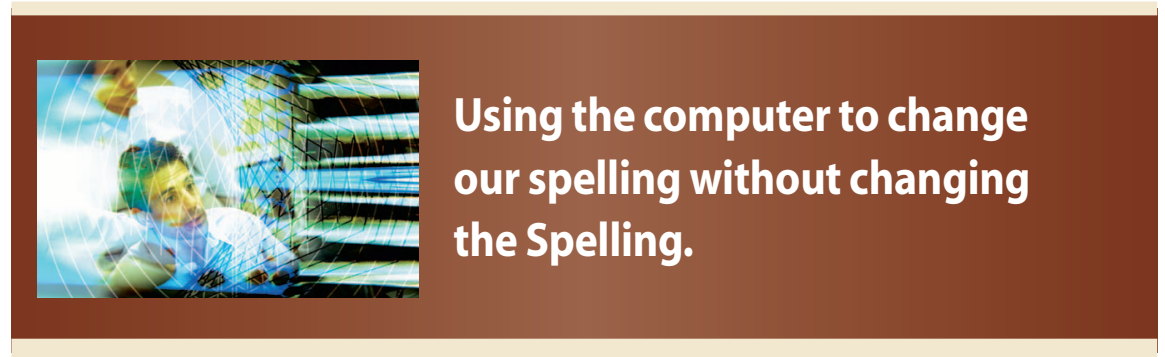

T he recent controversy in England over the government's proposal to teach students how to read using the phonics approach instead of the whole-word approach (http://news. bbc.co.uk/1/hi/education/4667518. stm) highlights the difficulties children have when learning to read English. Spelling also presents obstacles to the teaching of spoken English as a second language, in particular when students from developing countries want to study in English-speaking countries' colleges or universities.

The basic problem is the mismatch between how English is spoken and written. No other language that uses the Latin alphabet suffers from this problem to anything like the same degree, French's well-known foibles being relatively minor and systematic. In languages like Finnish and Italian, the spelling of words specifies very closely how they should be said, at least in the official version of the spoken language. This correspondence between writing and speech is maintained in many languages by occasional official spelling reforms, in Dutch and German for example, although the 1996 German reform didn't go over very well and the present rereform has met with some skepticism (www.dw-world.de/ dw/article/0,,1922563,00.html).
There have been attempts at spelling reform for English (en.wikipedia.org/ wiki/Spelling_reform). The only successful one, by Noah Webster well over a century ago, led to American/ British alternatives like ax/axe, fiberl fibre and plow/plough, but these are quite minor. More recently, the Australian federal government in the 1970s quite briefly and very half-heartedly adopted Harry Lindgren's staged SR1 approach (http://spellingsociety. org/journals/j9/sr1.php).

More radical and idealistic was George Bernard Shaw's espousal of a completely new alphabet just for English, but the contest for such an alphabet arising from his will led only to his Androcles and the Lion being published bimodally. Sir James Pitman's Initial Teaching Alphabet was popular for a while as a way to teach reading to beginning classes, but the transition from ITA to normal reading proved too hard for most (http://news. bbc.co.uk/1/hi/uk/1523708.stm).

There are two main barriers to English spelling reform. First, there is a great variety of different versions of spoken English, a splendid variety held together by the common spelling. Second, there is an enormous stock of written English made useful by the loose coupling of spelling and speech, and by online and offline readers' dependence on dictionaries and the like, that spelling reform would make difficult to use.

The widespread use of digital technology nowadays opens a way past these barriers. That way is quasireform: changing the spelling without changing the Spelling, by showing or suggesting the pronunciation typographically and variably.

\section{ANALOGIES}

Aspects of pronunciation can be marked by the written rendering of letters analogous to their rendering in speech, as Figure 1 shows.

\section{Hue}

A letter or group of letters can often be pronounced in two different ways. Within phrases or polysyllabic words, it is usual to have a pattern in which the vowels of the stressed syllables are more heavily pronounced. This can be marked by heavy coloring or, as in the following examples, by darkening the vowel concerned, as Figure 1 (a) shows.

The same technique can be used on consonants: normal for their nominal pronunciation and dark for their less usual variations, as Figure 1 (b) shows. Letters such as $\mathrm{c}$ and $\mathrm{g}$ frequently need darkening, others less so. Often, the context suggests using the alternative, but exceptions are so frequent, it's best to strictly mark the exception by darkening.

What can be darkened can also be lightened. It's appropriate to make letters lighter if they are not pronounced at all, as Figure 1 (c) shows, or if they are not needed to show the pronunciation, as Figure 1 (d) shows.

\section{Grouping}

Groups of letters are often pronounced as a single sound. This grouping can be shown by moving letters in the group closer together.

In English, pairs of letters represent many consonantal sounds. This can be indicated by moving the letters closer together and darkening them to show an alternative, as Figure 1 (e) Continued on page 102 
(a)

content with the content

to extract an extract

(b)

can cent of fob get gem in ink his sit

(c)

head heart heir ghost give history

knot off psychic torque wren

(d)

back edge egg ghetto guest hell singe

(e)

chat chef thick this cough

finger singer graph rough

(f)

fain bean mœaning doubtful schœol tœe

(g)

bar hair hear calm core fir harm paw

(h)

buy cow eye flay hourly sew toy

(i)

altar any foot only put

(k)

bitten bottle double brOther wOman women

(m)

attend forget lament petard posess

(n)

beat beet pair pear raid rein tart taut

Figure 1. Aspects of pronunciation can be marked by the written rendering of letters analogous to their rendering in speech, as examples (a) through (n) show.

shows. The same can be done for vowel groups, as Figure 1 (f) shows, although these might need further marking. Other letters should also be closed up with vowels when they lengthen their pronunciation, as Figure 1 (g) shows, or to make diphthongs of them, as Figure $1(\mathrm{~h})$ shows.

\section{DISTINGUISHING VOWELS}

Simple darkening or lightening applies to grouped or separated vowels or grouped or separated consonants. These conventions go far toward showing pronunciation, but might not go far enough for effective teaching. Vowels, particularly when long or diphthongal, are much more the vehicle of regional accents than consonants. This will make further marking controversial, but some schemes can be suggested.

\section{Short vowels}

Marking these presents two problems. First and least is that sometimes speakers give a group a short pronunciation. Although this can be dealt with by lightening all but one of the group, the major problem is that English has seven short vowels and only five vowel symbols. The following diagram shows six short vowels arranged to suggest where in the mouth they are pronounced.

$$
\begin{aligned}
& \text { pit put } \\
& \text { pet pot } \\
& \text { pat putt }
\end{aligned}
$$

The seventh short vowel is neutral, like the first vowel in attend, petard, petite, and petunia, and only occurs in unstressed syllables. The vowels in the top line are spoken with the mouth much more closed than for those in the bottom line, and the vowels in the words on the left are pronounced toward the front of the mouth, the others toward the back.

The diagram reflects the usual short pronunciation of the five vowel symbols. The vowel in "put" has no usual letter. When the short vowel is isolated and pronounced as usual, no marking is needed, but there are many exceptions. Horizontal displacement in the mouth can be marked by horizontal shifting of the vowel, and vertical displacement likewise, as Figure $1(\mathrm{j})$ shows. The neutral vowel can be shown as light and coupled to an adjacent semivowel, as Figure 1 (k) shows, or light and lifted otherwise to suggest an apostrophe, as Figure 1 (m) shows.

\section{Other vowels}

Marking more complex vowels will be both controversial and complicated. My suggestions here are thus tentative indeed. Long vowels can be construed as repeating the pattern of short vowels, with the long neutral vowel as in err.

$$
\begin{gathered}
\text { ear ooh } \\
\text { air ore } \\
\text { eh are }
\end{gathered}
$$

The eh is only very roughly analogous to the short a, and the long vowel shown as ooh is that of suit and two. The spellings for these are irregular. 
When the spelling is a group, the pronunciation can be marked by treating the group in the same way as the short vowel, although the vowels in the group might need to be marked differentially to highlight the one conveying the pronunciation, as Figure 1 (n) shows. Diphthongs can be similarly marked but distinguished from long vowels by moving the letters in the group vertically so that they no longer align horizontally.

There are other complications. Sometimes long vowels and diphthongs are spelled with a single letter. Such long vowels could be marked by widening the letter or by having the superposed dot of an i become a macron: $\bar{i}$. Such diphthongs could be marked perhaps by oriented distortion. Sometimes a letter $\mathrm{e}$ is used to complicate a preceding but not adjacent vowel, as in dune or fine.

Any final solution will likely be somewhat simpler than my incomplete suggestions and must compatibly deal with uppercase marking. The essential principle is that while the traditional spelling remains clear, the individual letters are manipulated. This lets programs provide variability in strength of marking, another essential aspect of quasireform.

\section{PARAMETRIC VARIABILITY}

Modern digital technology not only enables markings that suggest pronunciation but also lets their strength be varied.

Latin alphabet browsers and document formatters or printer drivers could let the user specify how strongly the pronunciation should be marked or whether to have it marked at all. Drill and practice programs for teaching reading of English, which I believe would be much more effective in instilling literacy in English-speaking elementary schools than any solely teacher-delivered method, could adjust the strength of marking automatically to suit the individual learner. The aim would be to move the learning reader smoothly from marked reading to unmarked in a way that ITA couldn't manage, although it is similar in other ways.
The marking could also be used in ordinary English text to show variant pronunciations, to mark the pronunciation of personal names and of new and borrowed words, to store recorded speech textually, and to mediate speech synthesis. This means that some marking would have to be fixed and some variable. Marking for international spoken English could be done automatically for most words, but there would always be a need to override this.

Two technical needs follow. The first is for a standard encoding of Latin alphabetic text that would facilitate quasireform (see "Sortemic Text Encoding" in my forthcoming book, Computers and People, Wiley, 2006). The extreme difficulty of composing the examples for this essay underscores the need for an encoding of English that treats the Latin alphabet as a graphical system-a difficulty that forced their separation from the text itself. Such an encoding would have many advantages other than its support for quasireform, and not just for English (see my "Toward Decent Text Encoding," Computer, Aug. 1998, pp. 108-109). One way to introduce such a system would be as another 8-bit subset of Unicode.

The second and consequent need is for a standard Latin alphabet keyboard to make such encoding simple.

\section{INTRODUCING QUASIREFORM}

This description of quasireform shows only its nature and practicality. The details reflect my particular view of how people speak unaccented English-different interpretations will likely be more practical or widely acceptable. Also, while I have described the marking techniques I think most suitable, diacritical marking offers another possibility. I didn't use it because I feel it should be reserved for use with names and borrowed words from languages that use them in their own ways, like the accents in French and the tone marking in romanized Chinese.

Before any such reform could be introduced, we would need an inter- national agreement among teachers, typographers, phoneticians, and computing professionals on just what system of marking should be used. This would take into account the different needs of elementary school pupils learning to read and of students learning to speak English as a second language.

\section{Q} uasireform of English spelling is particularly and urgently significant to the computing profession for two reasons. First, English has become the lingua franca of the international computing profession and industry to an even greater degree than in possibly any other important area, even that of international diplomacy and politics.

Second, the decline of literacy and numeracy in First-World Englishspeaking countries has led to the increasing dependency of their computing industries and formal computing education on immigrant professionals. The reasons for this go beyond the difficulty of learning to read and reflect the change in social culture from rational and contemplative values to impulsive and sensual ones. Nonetheless, no balance between these aspects of culture can be restored without restoration of basic conversational, literary, and numeric skills.

Quasireform will be difficult to sell, develop, and implement. The participation of the computing profession in all these phases would be crucial, but with the undertaking being so technically and politically complex, it is even more crucial that this participation not be seen as dominant. Computing professionals should be the facilitators of other professions' work-partners, not bosses.

Neville Holmes is an honorary research associate at the University of Tasmania's School of Computing. Contact him at neville.holmes@utas.edu.au.Details of citations in this essay, and links to further material, are at www.comp.utas. edu.au/users/nholmes/prfsn. 